\title{
Maternal embryonic leucine zipper kinase is a novel target for proliferation-associated high-risk myeloma
}

\author{
Arnold Bolomsky, ${ }^{1 \#}$ Roy Heusschen, ${ }^{2 \#}$ Karin Schlangen, ${ }^{3}$ \\ Kathrin Stangelberger, ${ }^{1}$ Joséphine Muller, ${ }^{2}$ Wolfgang Schreiner, ${ }^{3}$ \\ Niklas Zojer, ${ }^{1}$ Jo Caers ${ }^{2,4^{*}}$ and Heinz Ludwig ${ }^{1 *}$
}

${ }^{1}$ Wilhelminen Cancer Research Institute, Department of Medicine I, Wilhelminenspital, Vienna, Austria; 'Laboratory of Hematology, GIGA-I3, University of Liège, Belgium; ${ }^{3}$ Center for Medical Statistics, Informatics and Intelligent Systems, Section for Biosimulation and Bioinformatics, Medical University of Vienna, Austria and ${ }^{4}$ Division of Hematology, Department of Medicine, University and CHU of Liège, Belgium

$\# A B$ and $\mathrm{RH}$ are co-first authors. ${ }^{*} \mathrm{C}$ and $\mathrm{HL}$ contributed equally to this work.
Haematologica 2018

Volume 103(2):325-335

\section{ABSTRACT}

1 reatment of high-risk patients is a major challenge in multiple myeloma. This is especially true for patients assigned to the gene expression profiling-defined proliferation subgroup. Although recent efforts have identified some key players of proliferative myeloma, genetic interactions and players that can be targeted with clinically effective drugs have to be identified in order to overcome the poor prognosis of these patients. We therefore examined maternal embryonic leucine zipper kinase (MELK) for its implications in hyper-proliferative myeloma and analyzed the activity of the MELK inhibitor OTSSP167 both in vitro and in vivo. MELK was found to be significantly overexpressed in the proliferative subgroup of myeloma. This finding translated into poor overall survival in patients with high vs. low MELK expression. Enrichment analysis of upregulated genes in myeloma cells of $M E L K^{\text {high }}$ patients confirmed the strong implications in myeloma cell proliferation. Targeting MELK with OTSSP167 impaired the growth and survival of myeloma cells, thereby affecting central survival factors such as MCL-1 and IRF4. This activity was also observed in the 5TGM.1 murine model of myeloma. OTSSP167 reduced bone marrow infiltration and serum paraprotein levels in a dose-dependent manner. In addition, we revealed a strong link between MELK and other proliferation-associated high-risk genes (PLK-1, EZH2, FOXM1, DEPDC1) and MELK inhibition also impaired the expression of those genes. We therefore conclude that MELK is an essential component of a proliferative gene signature and that pharmacological inhibition of MELK represents an attractive novel approach to overcome the poor prognosis of high-risk patients with a proliferative expression pattern.

\section{Introduction}

The implementation of novel treatment opportunities have continuously improved the outcome of multiple myeloma (MM) patients throughout the last decades. ${ }^{1}$ However, clinical progress is mainly based on superior outcome in standard-risk patients, while the outcome in high-risk patients is still limited. ${ }^{2,3}$ Deciphering gene networks and drug candidates in high-risk $\mathrm{MM}$, in order to improve the prognosis of all MM patient subgroups, remains a major task.

Common classifications use tumor load and the presence of fluorescence in situ hybridization (FISH)-determined cytogenetic aberrations to define high-risk patients. ${ }^{4}$ More sophisticated methods include flow cytometry and gene expression profiling (GEP) to characterize patients with poor prognosis. ${ }^{5-10}$ The latter enabled the classification of MM into distinct GEP-defined subgroups. ${ }^{10}$ These subgroups are typically linked to the cytogenetic profile of $\mathrm{MM}$ (presence of distinct immunoglobulin heavy chain $[\mathrm{IgH}]$ translocations or hyperdiploidy). However, GEP studies also elucidated a proliferation-associated subtype. ${ }^{10}$ GEP-defined

\section{Correspondence: \\ heinz.ludwig@aon.at/jo.caers@chu.ulg.ac.be}

Received: May 17, 2017.

Accepted: October 27, 2017.

Pre-published: November 9, 2017.

doi:10.3324/haematol.2017.172973

Check the online version for the most updated information on this article, online supplements, and information on authorship \& disclosures: www.haematologica.org/content/103/2/325

\section{(C)2018 Ferrata Storti Foundation}

Material published in Haematologica is covered by copyright. All rights are reserved to the Ferrata Storti Foundation. Use of published material is allowed under the following terms and conditions:

https://creativecommons.org/licenses/by-nc/4.0/legalcode. Copies of published material are allowed for personal or internal use. Sharing published material for non-commercial purposes is subject to the following conditions:

https://creativecommons.org/licenses/by-nc/4.0/legalcode, sect. 3. Reproducing and sharing published material for commercial purposes is not allowed without permission in writing from the publisher. 
myeloma with a proliferative character is strongly associated with high-risk scores and, consequently, poor prognosis. ${ }^{8-10}$ Importantly, the outcome of this patient subgroup remains poor with current treatment strategies and thus requires the implementation of more specialized treatment approaches to improve survival rates.

Recent efforts have identified several key proliferative genes in MM. Among others, aurora kinase A (AURKA), ${ }^{11}$ polokinase-1 (PLK1), ${ }^{12,13}$ pituitary tumor transforming gene $1(\text { PTTG1 })^{14}$ and DEP domain containing 1a (DEPDC 1A $)^{15}$ overexpression has been reported in proliferative $M M$, and linked to poor prognosis. Targeting of these genes impaired the growth and survival of MM cells, but their functional relevance for the proliferative character in $\mathrm{MM}$ is unclear. Moreover, information about interactions or the hierarchy of individual candidate genes is limited at the moment. In this context, FOXM1 was recently reported to be a putative driver in high-risk $\mathrm{MM} .{ }^{16} \mathrm{~A}$ close relationship between FOXM1, CDK6 and NEK2 suggested a functional role for this transcription factor in promoting high-risk disease. CDK6 and NEK2 are transcriptional targets of FOXM1, and co-regulation of FOXM1 with these genes was linked to poor outcome. In addition, physical interaction between CDK6 and FOXM1 was suggested to further promote FOXM1-mediated gene transcription. ${ }^{16}$ However, the central drivers of proliferation-associated high-risk $\mathrm{MM}$ remain undiscovered and clinical grade inhibitors for many recently characterized target genes (e.g., FOXM1) are missing.

MELK, a serine/threonine kinase with strong implications in cell cycle regulation, ${ }^{17,18}$ was identified as an upstream regulator of FOXM1 in solid and hematological malignancies. ${ }^{19,20}$ MELK plays a functional role during cell cycle progression via a direct interaction with CDC25B and co-localization with key proteins such as cyclin B1 and CDK1. ${ }^{17}$ Overexpression of MELK as well as an association between MELK levels and poor prognosis has been reported in various malignancies. ${ }^{21-26}$ MELK was shown to play a role in the proliferation and survival of malignant cells and to support the growth of cancer stem cells. ${ }^{27-29}$ Mechanistically, MELK was found to regulate FOXM1 mediated expression of mitotic genes in a PLK1-dependent manner in glioblastoma and to induce EZH2 expression in irradiation-resistant glioma stem cells. ${ }^{19,30}$ More recent studies revealed additional MELK targets (e.g., DEPDC1), and demonstrated disruption of the MELK-associated gene network by using the MELK inhibitor OTSSP167 in solid and hematological malignancies. ${ }^{20,31,32}$ These reports placed MELK upstream of several genes independently linked to high-risk myeloma, including FOXM1, EZH2, PLK1 and DEPDC1. ${ }^{12,13,15,16,33}$ Considering the availability of a MELK small molecule inhibitor (OTSSP167) already undergoing clinical testing, ${ }^{34}$ we aimed to analyze the role of MELK in high-risk MM.

\section{Methods}

See the Online Supplementary Materials and Methods for a description of the techniques.

\section{Cells}

Human multiple myeloma cell lines (HMCLs) U266, KMS-12BM, OPM-2, NCI-H929, SK-MM-1, RPMI8226, MM.1S, and MM.1R as well as immortalized bone marrow (BM) mesenchymal stromal cells (kindly provided by Dr. Dario Campana, St. Jude Children's Research Hospital, Memphis, TN, USA) were cultivated as previously described. ${ }^{35}$ TTGM.1GFP+ cells (kind gift of Dr. G. Mundy, Vanderbilt University, Nashville, TN, USA) and HEK293T cells were maintained in Dulbecco's modified eagle medium (DMEM), supplemented with 10\% fetal bovine serum (FBS), $2 \mathrm{mM} \mathrm{L-glutamine} \mathrm{and} \mathrm{1 \%} \mathrm{penicillin/streptomycin.}$

\section{Cytotoxicity and colony formation assays}

Cytotoxicity and colony formation assays were performed using Cell Counting Kit 8 (Sigma-Aldrich) and MethoCult Classic methylcellulose based medium (Stem Cell Technologies) as described previously. ${ }^{35}$

\section{Flow cytometry}

All assays were performed according to the manufacturer's instructions as described previously. ${ }^{35}$ Analyses were performed on a FACScan and FACS Canto II (BD Biosciences).

\section{In vivo study}

For studies in the murine 5TGM.1 myeloma model, OTSSP167 was dissolved in $0.5 \%$ methylcellulose (Sigma-Aldrich) and administered by oral gavage at different dose levels. For every experimental cohort, mice were randomly divided into a naïve group ( $\mathrm{n}=5$, healthy controls), a vehicle group ( $\mathrm{n}=10$, myelomabearing mice receiving vehicle solution) and a treated group ( $n=10$, myeloma-bearing mice receiving OTSSP167). These experiments were performed as previously described. ${ }^{36}$ The Institutional Animal Care and Use Committee (ICACUC) approval number is 1336. The accreditation number from the Belgian government is LA16100002/LA2610359.

\section{Statistical analysis}

Patient groups exhibiting higher or lower target gene expression were defined with the maximally selected rank statistics, implemented in the maxstat $R$ package. Statistical significance of differences in overall survival (OS) was calculated by the log-rank test, and survival curves were plotted using the Kaplan-Meier method. Pearson correlations were calculated using $\mathrm{R}^{\prime}$ s cor.test function. For the analysis of in vitro and in vivo experiments, a two-tailed unpaired $t$-test was performed for the comparison of 2 means and one-way ANOVA followed by a Tukey's post hoc test for comparison of multiple means by Prism 5 (GraphPad Software Inc., La Jolla, CA, USA). $P$-values $<0.05$ were considered to be statistically significant. Drug combinations were analyzed with CompuSyn software. Combination index (CI) values $<0.85,0.85-1.15$, and $>1.15$ were interpreted as synergistic, additive, and antagonistic drug activity, respectively. All graphs represent the mean \pm standard deviation of at least three independent experiments performed in triplicates unless otherwise indicated.

\section{Results}

MELK expression is elevated in proliferation-associated high-risk myeloma and linked to poor outcome

To study the clinical relevance of MELK in MM, we analyzed MELK gene expression levels in publically available GEP datasets. No significant difference was observed between gene expression levels of healthy donor bone marow plasma cells (BMPCs), monoclonal gammopathy of undetermined significance (MGUS) and smoldering multiple myeloma (SMM) patient cells, but we noted a stepwise increase from MGUS/SMM cells to newly diagnosed and relapsed myeloma (Figure 1A). Analysis of MELK expression in distinct GEP-defined subgroups 
(according to Zhan et al. ${ }^{10}$ ) revealed significant overexpression of $M E L K$ in newly diagnosed patients categorized into the proliferation (PR) subgroup of MM (Figure 1B). Consequently, high MELK expression levels were associated with poor outcome in patients treated within the total therapy 2 (median OS not reached vs. 81.47 months, $P=0.01$ ), total therapy 3 (median $O S$ not reached, $P<0.0001)$ as well as bortezomib- and/or dexamethasonebased protocols (median OS 21.1 months vs. 11.2 months, $P=0.02$ ) (Figure 1C). A similar association was noted with PANP-defined detectable and absent MELK expression as cut-off (Online Supplementary Figure S1). Moreover, comparison of MELK expression levels in MM patients at baseline vs. relapse indicated significant $M E L K$ upregulation in CD138-purified BM cells of relapsed patients, suggesting selection of $M E L K^{\text {high }} \mathrm{MM}$ cells or increasing MELK expression upon treatment which could be implicated in drug resistance (Figure 1D). To further strengthen the link between MELK and high-risk disease we analyzed MELK expression levels using independent GEP-datasets which contained samples from patients with plasma cell leukemia (PCL). This clearly demonstrated an upregulation of $M E L K$ in PCL compared to $\mathrm{MM}$, underlining the strong association between MELK expression and aggressive disease (Figure 1E,F).

\section{MELK expression is strongly associated with cell cycle regulation}

In order to confirm the association of MELK with proliferation in MM, we analyzed GEP data (GSE24080) of newly diagnosed $\mathrm{MM}$ patients $(\mathrm{n}=551)$ with high vs. low levels of MELK. This depicted 266 upregulated and 5 downregulated probe sets representing 235 genes (minimum fold-change $>2$ ) in patients with high compared to low MELK expression (Online Supplementary Table S1). High $M E L K$ expression levels were associated with an ele-

A

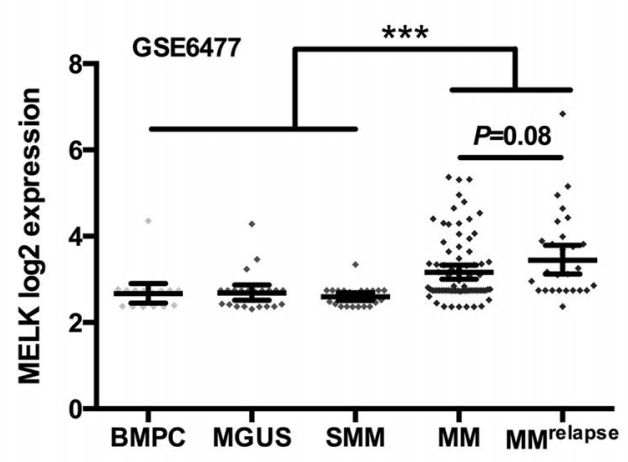

C
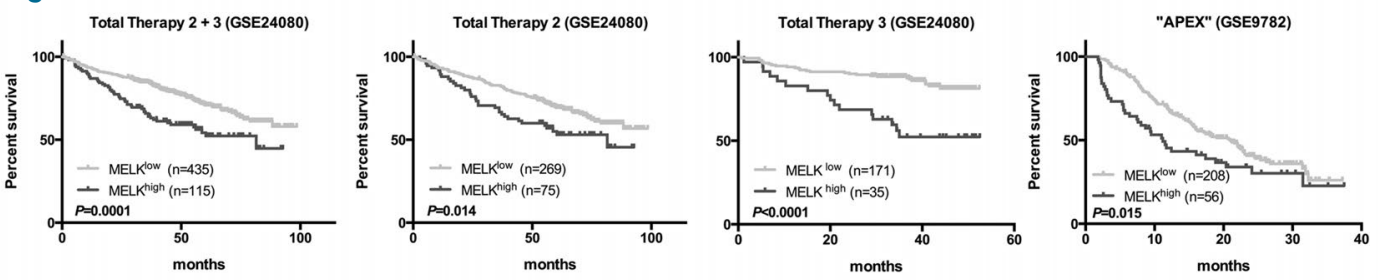

D

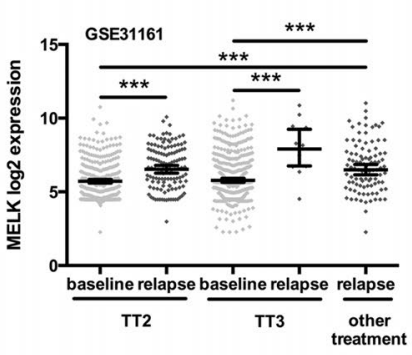

B

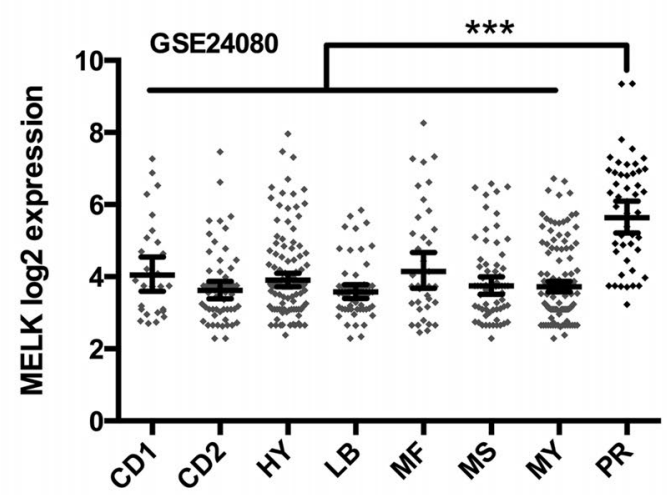

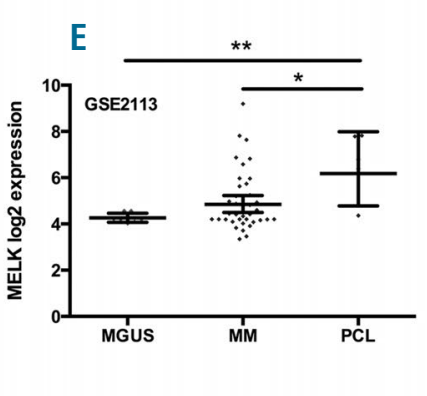

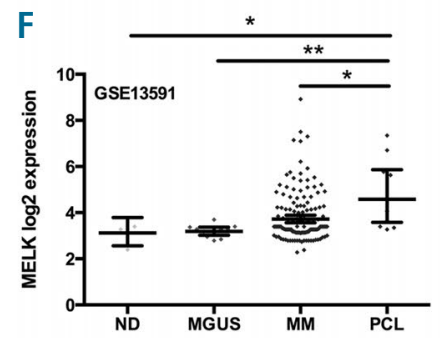

Figure 1. MELK is overexpressed in proliferation-associated myeloma and linked to poor outcome. (A) Analysis of publically available GEP data demonstrated significant overexpression of MELK in CD138+ purified cells of MM patients compared to MGUS and SMM patients as well as healthy donor bone marrow plasma cells (BMPCs). (B) Analysis of MELK expression in distinct GEP-defined subgroups revealed significant overexpression in the proliferation (PR)-associated subgroup of MM CD1: CCND1 group; CD2: CCND2 group; HY: hyperdiploid group; LB: low bone disease group; MF: Maf/MafB group; MS: MMSET group; MY: myeloid signature group. (C) High MELK expression was associated with poor outcome in newly-diagnosed patients treated within the total therapy 2 and 3 protocols (GSE24080) as well as relapsed and/or refractory patients (GSE9782) treated with bortezomib or dexamethasone. (D) MELK expression was elevated at relapse compared to baseline in patients treated within the TT2 ( $n=127$ and $n=343$, respectively) and TT3 ( $n=29$ and $n=453$, respectively) protocols as well as other treatment strategies ( $n=98)$. ( $E$ F) MELK expression was significantly elevated in $\mathrm{CD}_{138^{+}}$purified cells of patients suffering from PCL compared to BMPC, MGUS and MM cell samples. Horizontal lines indicate geometric mean with $95 \%$ confidence interval. $* P<0.05, * * P<0.01, * * * P<0.001$. PCL: plasma cell leukemia; MGUS: monoclonal gammopathy of undetermined significance; MM: multiple myeloma; SMM: smoldering multiple myeloma; ND: normal donor; TT: total therapy. 
vated transcription of several genes implicated in cell cycle regulation, such as CDK1, CCNB1, CCNB2, AURKA, KIF11, or $B U B 1 B$. This was confirmed by MetaCore enrichment analysis. The top-10 GO processes, pathway maps and process networks demonstrated a significant enrichment for cellular processes involved in cell cycle regulation (Online Supplementary Table S2). In brief, these results demonstrate a significant association of MELK with proliferation-associated high-risk myeloma, and therefore encouraged pre-clinical testing of MELK as a novel therapeutic target in MM.

Targeting of MELK impairs the growth and survival of myeloma cells

In line with their proliferative character, ${ }^{9}$ MELK messenger ribonucleic acid (mRNA) and protein expression was
A
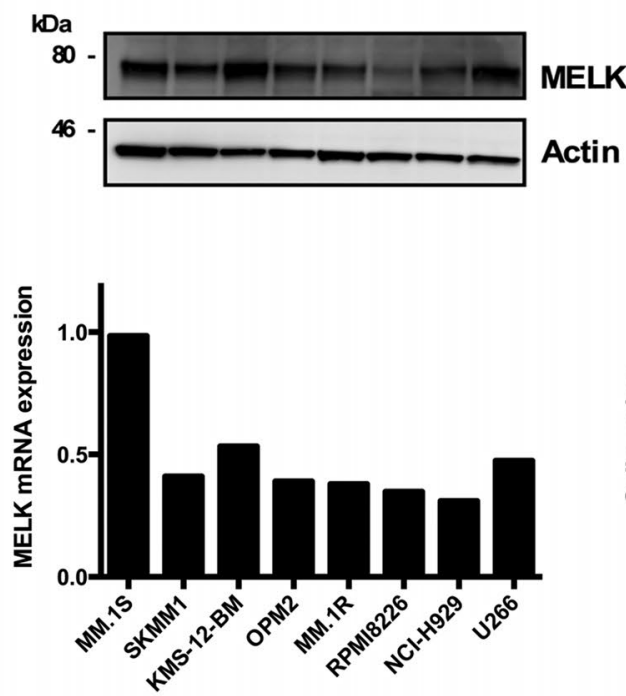

C

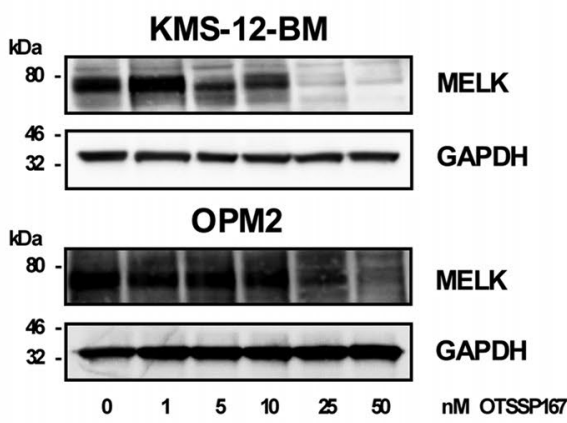

E

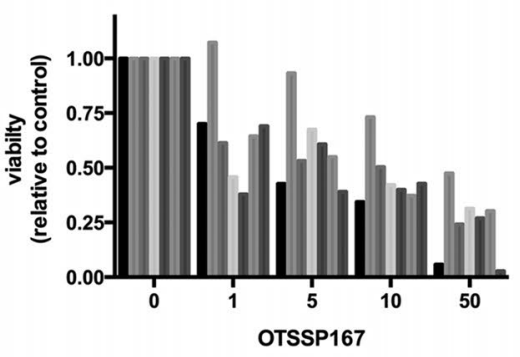

B
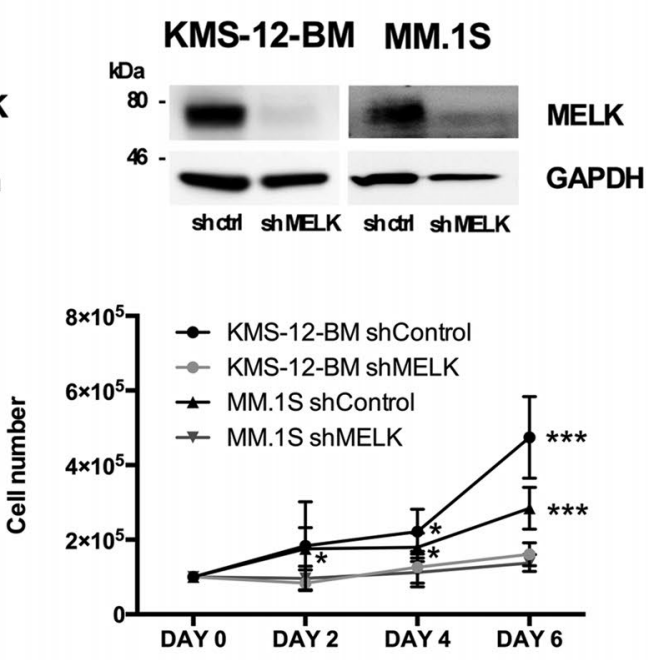

D

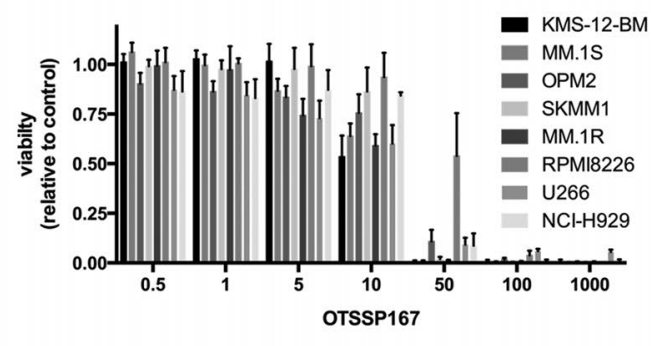

$\mathbf{F}$

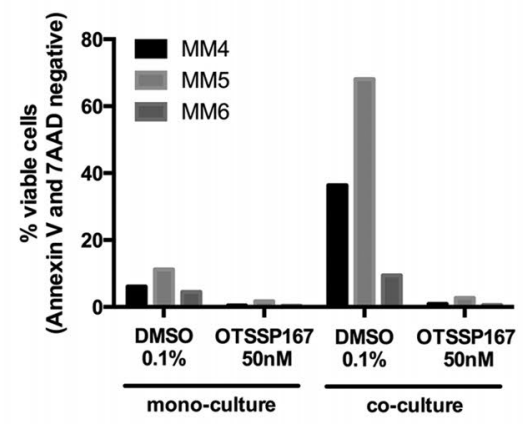

Figure 2. Targeting of MELK impairs myeloma cell growth. (A) MELK protein and mRNA expression in HMCLs. (B) KMS-12-BM and MM.1S cells transduced with MELK specific shRNA show significantly impaired cell growth compared to cells transduced with control vector carrying scrambled shRNA. $* P<0.05, * * * P<0.001$. (C) Treatment with OTSSP167 reduces MELK protein expression in HMCLs in a dose-dependent manner. Viability of (D) HMCLs and (E) primary MM cells 96 hours posttreatment with OTSSP167. (F) Viable primary MM cells were assessed by Annexin V/7-AAD staining 72 hours posttreatment with 0TSSP167 in the presence (coculture) or absence (mono-culture) of BSMCs. 
detected in all HMCLs analyzed (Figure 2A). The targeting of MELK via short hairpin (sh)RNA significantly impaired the growth of KMS-12-BM and MM.1S cells (Figure 2B). We therefore continued to study the impact of MELK inhibition on MM cells using a small molecule inhibitor of MELK (OTSSP167). Treatment with OTSSP167 leads to destabilization of MELK and a subsequent loss of MELK protein levels. ${ }^{31}$ Accordingly, OTSSP167 downregulated MELK protein levels 24 hours posttreatment and reduced the viability of all tested HMCLs (median IC50: $10.2 \mathrm{nM}$ range: $7.6-27.1 \mathrm{nM}$ ) (Figure 2C,D). Moreover, OTSSP167 showed similar activity in six out of seven primary MM cell samples obtained from patients with heavily pre-treated disease (Figure 2E; for patient characteristics see Online
A

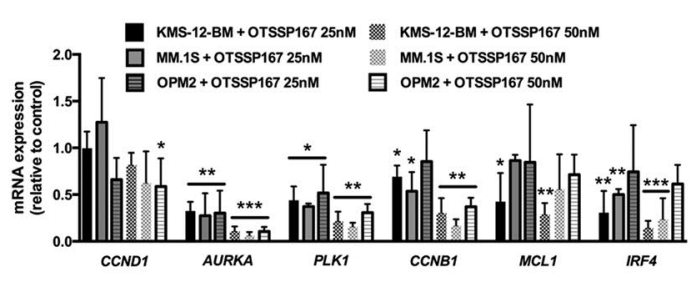

B

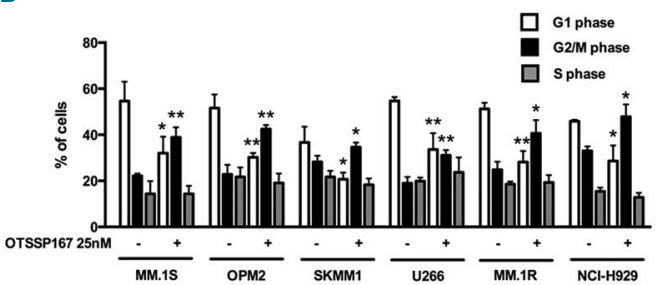

C

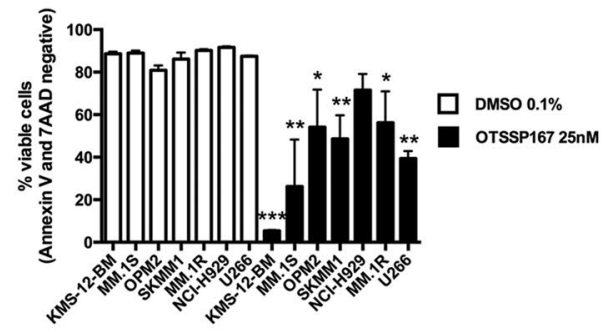

D

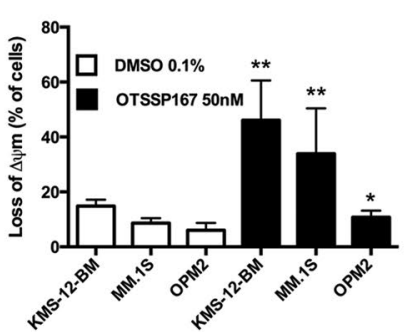

E
F

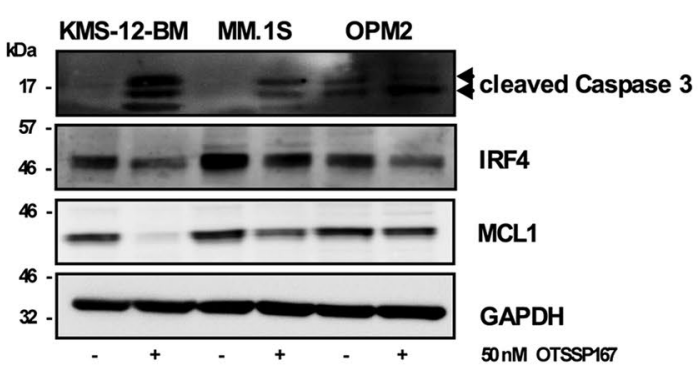

G

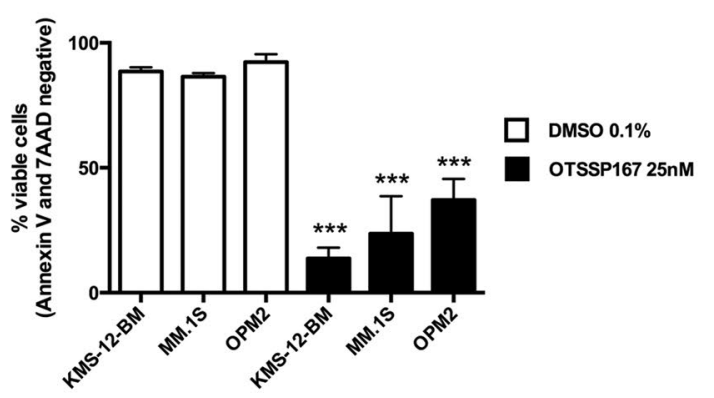

H

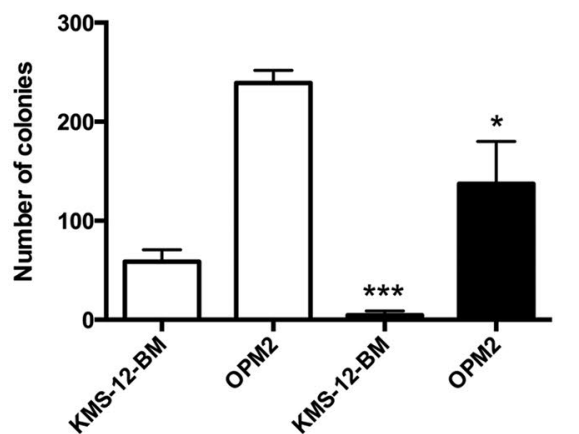

DMSO $0.1 \%$

OTSSP167 25nM

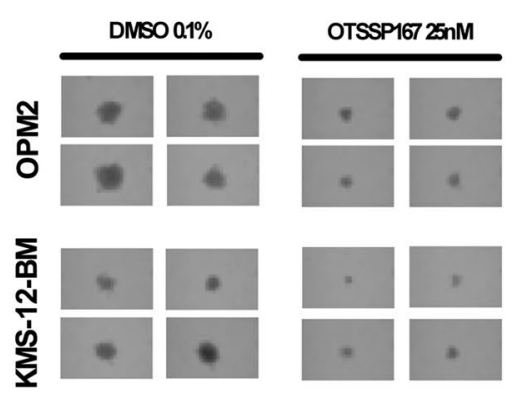

Figure 3. OTSSP167 impairs the cell cycle at the G2/M phase and induces apoptosis in MM cells. (A) Transcript levels of MM related growth and survival genes 5 hours posttreatment with OTSSP167 at the indicated concentrations. Gene expression levels are displayed relative to the control (0.1\% DMSO). (B) Cell cycle distribution of HMCLs 48 hours posttreatment with either 0.1\% DMSO (control) or OTSSP167. (C-E) Induction of apoptosis was verified by (C) Annexin V/7-AAD staining, (D) loss of the mitochondrial membrane potential, and (E) increased levels of cleaved PARP. (F) Representative Western blot images of HMCLs 24 hours posttreatment with either 0.1\% DMSO (control) or OTSSP167. (G) OTSSP167 induces apoptosis in MM cells in the presence of BMSCs. (H) OTSSP167 inhibited colony formation of HMCLs. Images are representative for three independent experiments. $* P<0.05, * * P<0.01$, and $* * * P<0.001$ compared to control. 
Supplementary Table S3). Due to the short-lived viability of in vitro propagated primary MM cells, we also analyzed the activity of OTSSP167 in co-culture with bone marrow stromal cells (BMSCs). In line with the reported supportive role of BMSCs this demonstrated a pro-survival effect on the viability of primary MM cells. Importantly, OTSSP167 completely abrogated the protective effect of BMSCs and eradicated viable MM cells obtained from patients with PCL or refractory MM (Figure $2 \mathrm{~F}$ ). In contrast, OTSSP167 displayed only a minor impact on the via- bility of human peripheral blood mononuclear cells (PBMCs) or BMSCs at effective anti-MM concentrations (median IC50: 726 nM) (Online Supplementary Figure S2).

Inhibition of MELK was accompanied by a rapid downregulation of central myeloma genes. In line with the proposed involvement of MELK in the G2/M phase of the cell cycle we observed reduced gene expression levels of CCNB1, AURKA and PLK1 5 hours posttreatment with OTSSP167 (Figure 3A). A significant correlation of MELK expression levels and those of CCNB1 $(\mathrm{R}=0.82$,
A

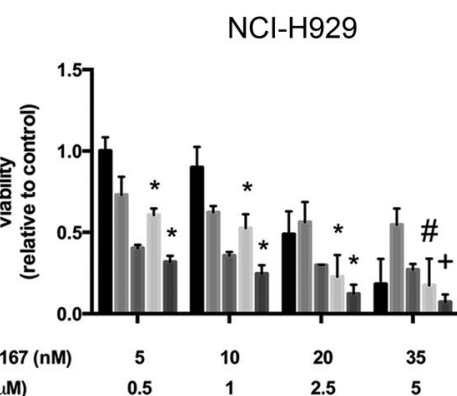

$\begin{array}{lcccc}\text { OTSSP167 (nM) } & 5 & 10 & 20 & 35 \\ \text { IMiDs }(\mu \mathrm{M}) & 0.5 & 1 & 2.5 & 5\end{array}$

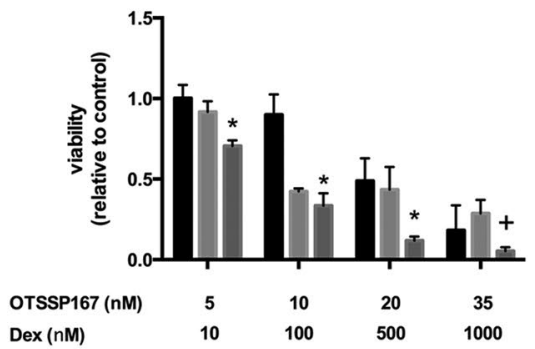

B

$\mathrm{NCl}-\mathrm{H} 929$
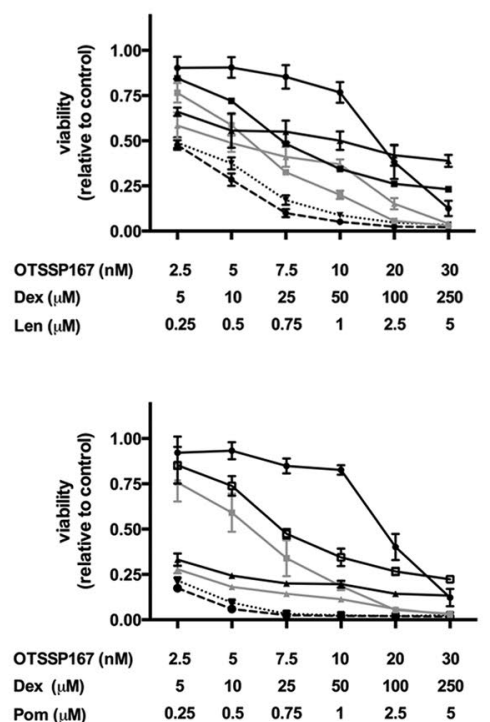

OPM2
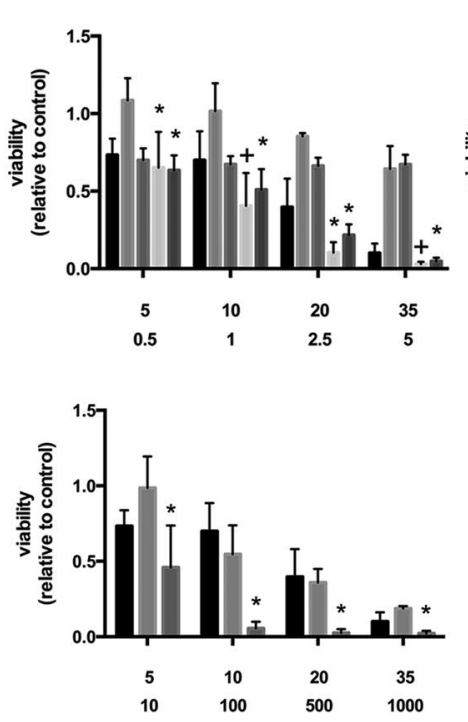

OPM2
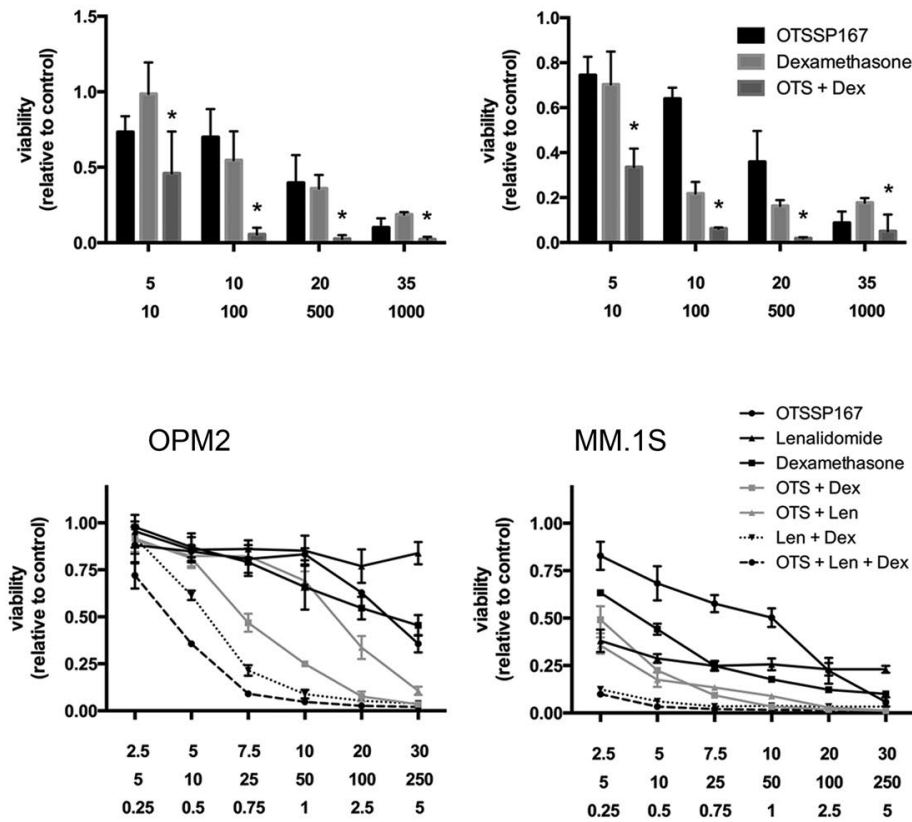

Figure 4. OTSSP167 displays synergistic activity with IMiDs and dexamethasone. (A) HMCLs were treated with OTSSP167 in combination with IMiDs (lenalidomide, pomalidomide) or dexamethasone for 96 hours at the indicated concentrations. $\mathrm{Cl}$ values were determined with CompuSyn. $\mathrm{Cl}$ values $<0.85,0.85-1.15$, or $>1.15$ indicate synergistic (*), additive (+) or antagonistic (\#) drug activity, respectively. (B) The activity of OTSSP167 in combination with dexamethasone plus lenalidomide or pomalidomide is compared to the corresponding monodrugs and dual-combinations. All data points of the triple combination in all three cell lines displayed strong synergism (Cls< 0.5 and 0.3 for lenalidomide and pomalidomide containing treatments, respectively; data not shown). OTS: OTSSP167; IMiDs: immunomodulatory drugs; Len: lenalidomide; Pom: pomalidomide; Dex: dexamethasone; Cl: combination index. 
$P<0.00001)$, AURKA $(\mathrm{R}=0.70, P<0.00001)$ and $P L K 1$ $(\mathrm{R}=0.38, P<0.00001)$ was noted in publically available GEP data (GSE24080) (data not shown). In addition, we observed a decreased expression of the prominent myeloma survival factors $M C L-1$ and IRF4 (Figure 3A). These findings translated into a significant accumulation of cells in the G2/M phase of the cell cycle as well as induction of apoptosis 48 hours and 72 hours posttreatment, respectively (Figure 3B,C). The latter was verified by a significant increase of AnnexinV/7-AAD positive cells (Figure 3C) and associated with a loss of the mitochondrial membrane potential, detection of cleaved PARP and cleaved caspase 3 (Figure 3D-F). Moreover, decreased expression of IRF4 and $M C L-1$ translated into reduced protein levels 24 hours posttreatment (Figure 3F).

Importantly, the anti-myeloma activity of OTSSP167 was upheld in the presence of BMSCs. Similar frequencies of apoptotic cells were observed in co-cultures compared to mono-cultures (Figure 3C and 3G). We also observed reduced clonogenic growth in OTSSP167-treated HMCLs (Figure $3 \mathrm{H}$ ). This additionally suggests an impact of MELK inhibition on tumor propagating cells.
0TSSP167 displays strong synergism with immunomodulatory drugs and dexamethasone

To examine a potential impact of OTSSP167 on the activity of established anti-myeloma drugs, we performed drug combination studies with immunomodulatory drugs (IMiDs; lenalidomide, pomalidomide), proteasome inhibitors (PIs; bortezomib, carfilzomib), dexamethasone, melphalan and bendamustine. These experiments demonstrated synergistic activity $(\mathrm{CI}<0.85)$ of OTSSP167 with IMiDs and dexamethasone, while combination studies with PIs displayed varying results. In detail, the combination of OTSSP167 with IMiDs displayed synergistic or additive drug activity in 22 of 24 combinations tested. Median CI values for lenalidomide and pomalidomide were 0.76 (range: 0.49-1.29) and 0.48 (range: 0.12-1.12), respectively. Strong synergistic activity of OTSSP167 was also observed in combination with dexamethasone. 11 out of 12 combinations were synergistic (one additive), with CI values ranging from 0.20-1.12 (median 0.43) (Figure 4A). In contrast, 13/21 evaluable combinations with PIs displayed antagonistic drug activity. Mainly additive effects were observed when OTSSP167 was com-
A
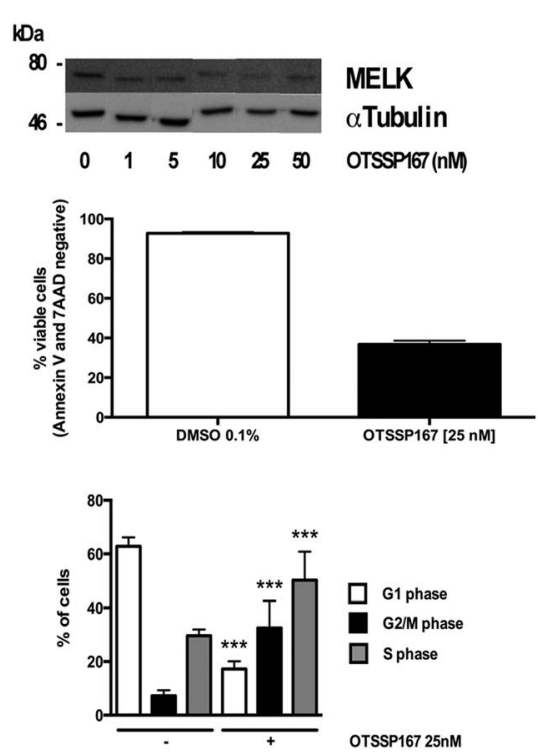

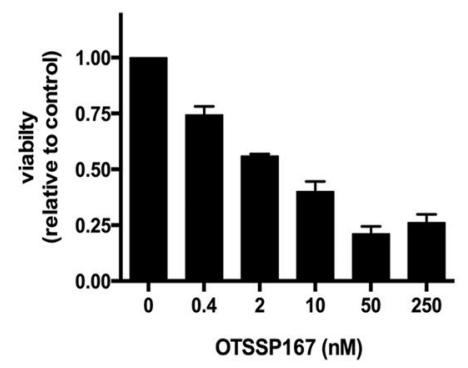

B i.v. $5 \times 10^{5} \mathrm{GFP}$
5TGM.1 cells

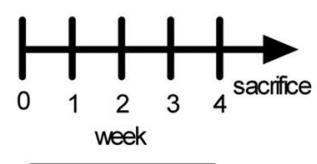

vehicle vs. OTSSP167
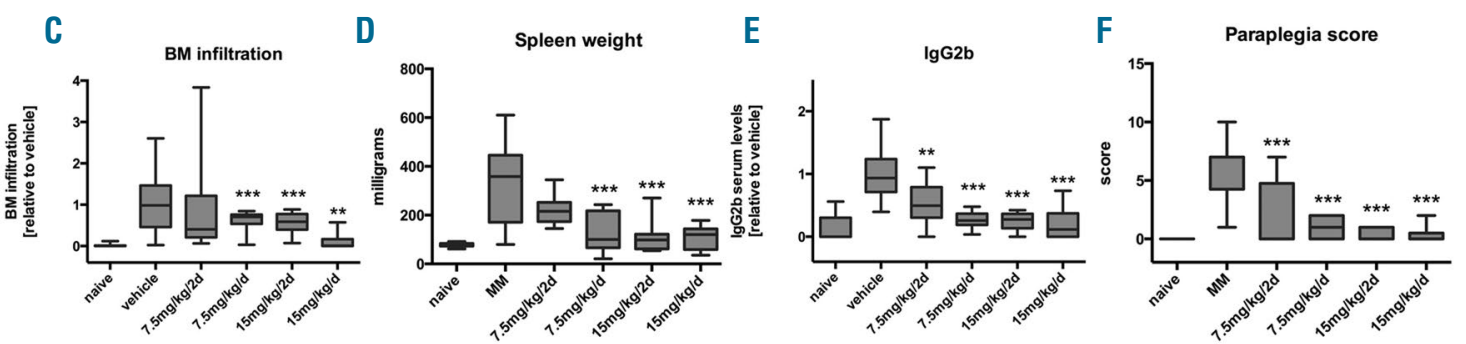

Figure 5. OTSSP167 impairs myeloma cell growth in vivo. (A) In vitro evaluation of OTSSP167 in murine 5 TGM.1 myeloma cells reduced MELK protein levels and cell viability in a dose-dependent manner 24 hours and 72 hours posttreatment, respectively. This was accompanied by a significant increase of cells in the G2/M phase of the cell cycle 24 hours after treatment initiation and accumulation of apoptotic cells 72 hours posttreatment $(P<0.001)$. (B) OTSSP167 treatment schedule in the 5TGM.1 murine model of myeloma. Treatment of myeloma bearing mice with OTSSP167 led to a dose-dependent reduction of (C) BMPC infiltration rate, (D) spleen weight and (E) serum IgG2b levels. In addition, the paraplegia score (based on presence and severity of paraplegia, altered posture and diminished activity) of OTSSP167-treated mice significantly improved compared to vehicle-treated mice (F). Box plots represent median (horizontal line) with min-max whiskers. $* * P<0.01$ and $* * * P<0.001$ compared to vehicle-treated control mice. BM: bone marrow; MM: multiple myeloma. 
bined with the alkylating drugs melphalan (median CI: 0.86 , range: $0.34-1.27)$ and bendamustine $(1.03,0.63-1.62)$ (Online Supplementary Figure S3).

We further confirmed the strong synergism between OTSSP167 and IMiDs as well as dexamethasone using a wider range of drug concentrations. This corroborated our results as OTSSP167 showed consistent synergism with IMiDs and dexamethasone independent of the concentrations used (Online Supplementary Table S4). Finally, we examined whether OTSSP167 is synergistic in combination with lenalidomide/pomalidomide plus dexamethasone. OTSSP167 displayed strong synergism with this well-established treatment regimen (median CI of OTSSP167 with lenalidomide-dexamethasone: 0.15, range: 0.02-0.46; pomalidomide-dexamethasone: 0.09 , range: $0.003-0.28)$. In spite of the potent combinatorial effect of IMiDs-dexamethasone, OTSSP167 further improved the efficacy of this combination; in particular in

A

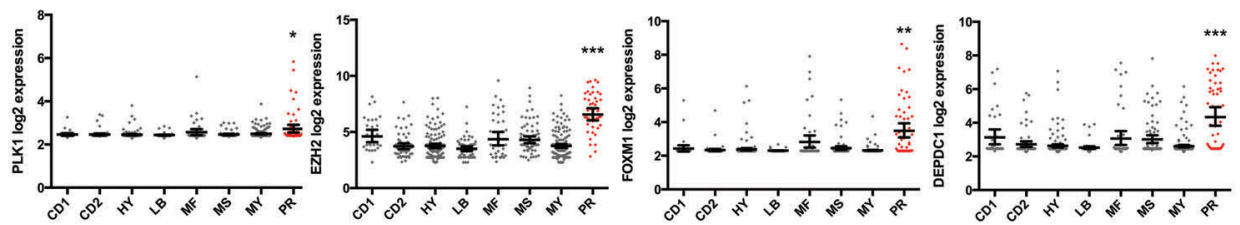

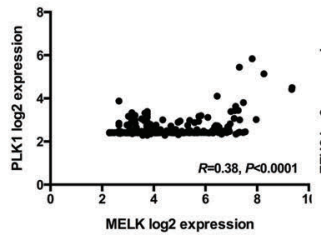
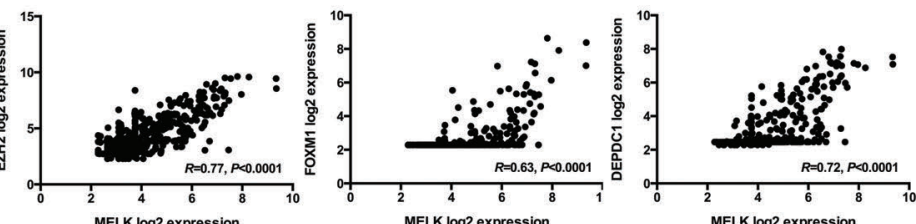

B
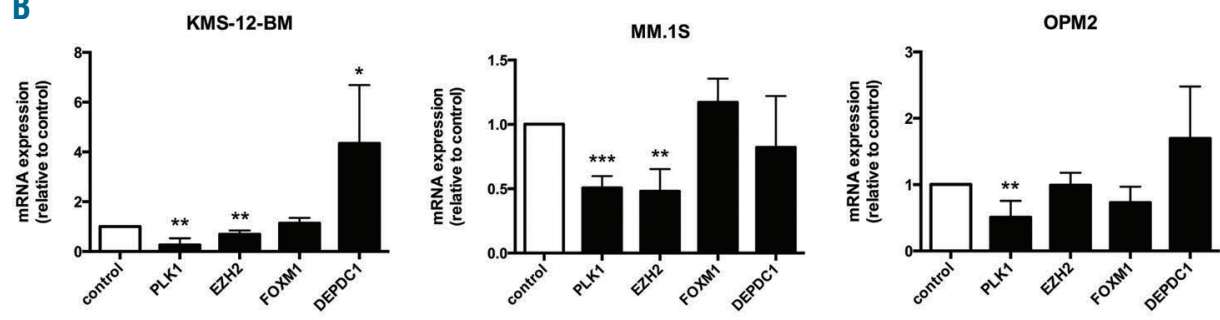

C

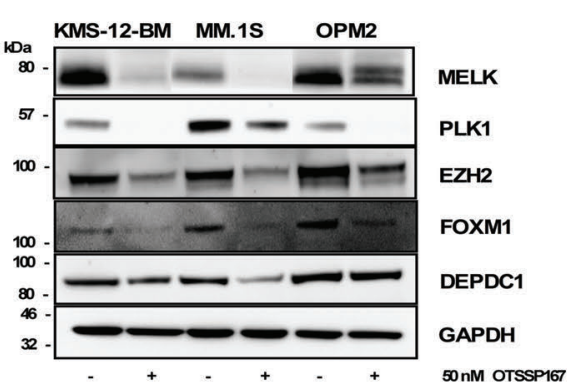

D

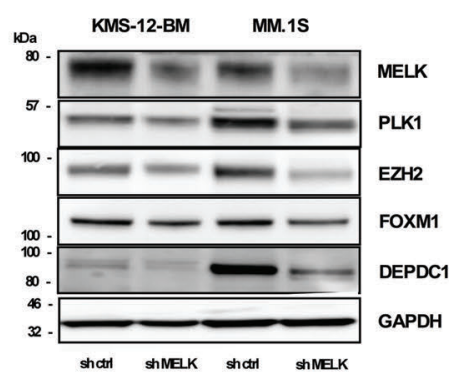

E

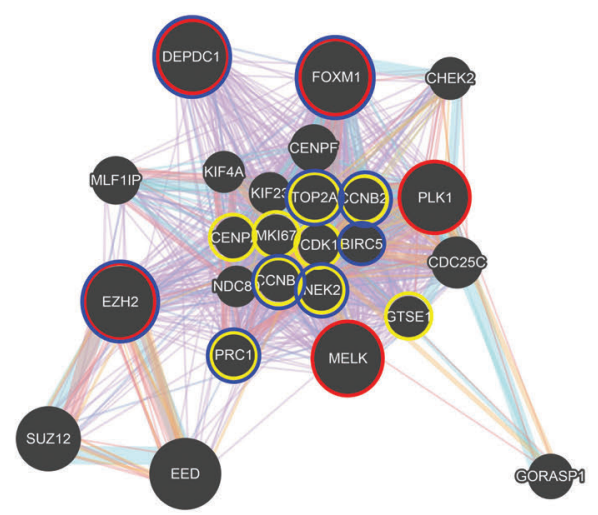

F

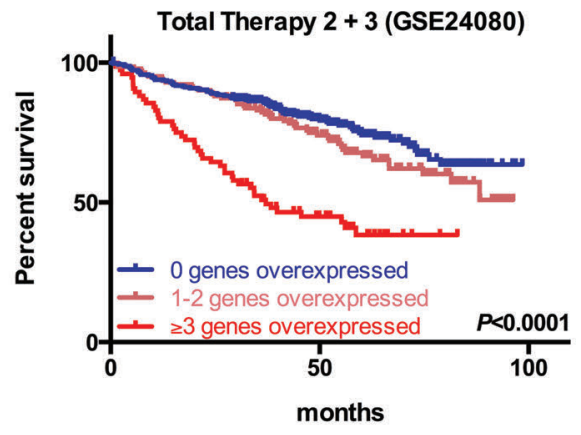

Figure 6. MELK is an essential component of a proliferationassociated high-risk network. (A) Gene expression levels of PLK1, $E Z H 2$, FOXM1 and DEPDC1 are significantly elevated in the proliferation (PR)-associated subgroup of myeloma $(* P<0.05, * * P<0.01$ and $* * * P<0.001$ compared to all other subgroups) and show a significant correlation with MELK expression levels. Horizontal lines indicate geometric mean with $95 \%$ confidence interval. (B) Gene expression levels of PLK1, EZH2, FOXM1 and DEPDC1 in HMCLs 24 hours posttreatment with OTSSP167. *P<0.05, **P<0.01 and $* * * P<0.001$ compared to control (0.1\% DMSO). (C, D) Protein expression of PR-associated high-risk genes (C) 24 hours posttreatment with OTSSP167 or (D) in cells transduced with a MELK specific shRNA. (E) The five genes, MELK, PLK1, EZH2, FOXM1 and DEPDC1 (marked in red) were used to reveal an underlying network of genes using GeneMANIA. The derived network included genes also found to be overexpressed in patients with high MELK expression (marked in yellow) as well as genes which are among the top 50 overexpressed genes of the PR subgroup of myeloma (marked in blue). ( $F$ ) Overall survival of newly-diagnosed MM patients treated within the total therapy 2 or 3 protocols based on MELK, PLK1, EZH2, FOXM1 and DEPDC1 expression. Patients were grouped according to overexpression of $0,1-2$ and $\geq 3$ of these PR-associated high-risk genes. CD1: CCND1 group; CD2: CCND2 group; HY: hyperdiploid group; LB: low bone disease group; MF: Maf/MafB group; MS: MMSET group; MY: myeloid signature group. 
OPM-2 and NCI-H929 cells at low doses with less pronounced activity of lenalidomide/pomalidomide plus dexamethasone (Figure 4B).

\section{TSSP167 impairs myeloma cell growth in vivo}

Treatment of murine 5TGM.1 MM cells with OTSSP167 demonstrated anti-myeloma activity similar to the effects observed in HMCLs. MELK transcript levels were in the range of HMCLs (data not shown), and we observed a dose-dependent reduction of MELK protein levels and viability 24 hours and 96 hours posttreatment, respectively. Moreover, OTSSP167 induced G2/M phase cell cycle arrest and apoptosis in 5TGM.1 cells (Figure 5A). This impact translated to the in vivo setting. OTSSP167 strongly reduced tumor burden in the 5TGM.1 murine model of myeloma. This was evidenced by several indicators including BM plasma cell infiltration, spleen weight and serum IgG2b levels (Figure 5B-E). We observed a dosedependent reduction of myeloma growth in the BM and spleen weight as well as normalization of paraprotein levels. In addition, OTSSP167 significantly enhanced the well-being of myeloma-bearing mice indicated by the absence of paraplegia and increased activity (Figure 5F).

\section{Inhibition of MELK impairs a proliferation-associated myeloma high-risk gene signature}

We next sought to decipher the relationship of MELK with other genes associated with high-risk disease. Similar to MELK, PLK1 is significantly upregulated in the GEPdefined PR subgroup of $\mathrm{MM}^{12}$ Other genes associated with poor outcome in $\mathrm{MM}$ and/or known functional ties with MELK include FOXM1, EZH2, and DEPDC1. ${ }^{15,16,37}$ Strikingly, all of these genes are significantly upregulated in the PR subgroup and associated with poor outcome. Correlation analysis confirmed a strong association between MELK expression levels and those of the other high-risk genes in MM cells (Figure 6A, Online Supplementary Figure S4). We therefore tested the impact of MELK inhibition on the expression of those genes. Treatment with OTSSP167 for a period of 24 hours led to a significant downregulation of PLK1 and EZH2 transcript levels (Figure 6B). Moreover, OTSSP167 downregulated PLK1, FOXM1, EZH2 and DEPDC1 protein levels in concert with MELK, suggesting a functional relationship of these genes in MM. This observation was confirmed with shRNA mediated MELK knockdown (Figure 6C,D).

To better understand the genetic network of these molecules and to reveal additional network partners, we analyzed the interactions of the five high-risk genes using GeneMANIA. This demonstrated close interactions of all five candidate genes in concert with several cell cycle-associated genes (Figure 6E). The top six annotated functions of this network were all linked to cell cycle regulation (cell cycle G2/M-phase transition, G2/M-phase transition of mitotic cell cycle, condensed chromosome kinetochore, nuclear division, condensed chromosome centromeric region, mitosis; false discovery rate $\left.<1.10 \times 10^{-9}\right)$. Of note, nine out of 20 proposed network genes (CENPA, PRC1, CCNB1, CCNB2, MKI67, TOP2A, CDK1, NEK2, GTSE1) were also found to be elevated in newly-diagnosed MM patients with high MELK expression (Online Supplementary Table S1), and nine genes of the proposed network plus input genes are among the top 50 overexpressed genes of the GEP-defined PR subgroup (DEPDC1, EZH2, FOXM1, NEK2, CCNB1, TOP2A, PRC1,
CCNB2, and BIRC5) (Figure 6E). The clinical relevance of this gene network was evident in patients treated within the total therapy 2 and 3 trials. Patients with elevated expression of three or more high-risk genes (MELK, PLK1, EZH2, FOXM1, DEPDC1) displayed a significantly shorter OS compared to patients with low expression levels of these genes, or only one or two genes with high expression (median OS 37.1 months vs. not reached, $P<0.0001$; Figure $6 \mathrm{~F}$ ). Taken together, these data strengthen our prior results and suggest a direct impact of MELK on other proliferation-associated genes as well as a functional role in proliferation-associated high-risk MM.

\section{Discussion}

The characterization of novel treatment opportunities for high-risk patients is a major task in current myeloma research. Herein, we identified MELK as a putative anti$\mathrm{MM}$ target in the proliferation-associated high-risk subgroup of $\mathrm{MM}$ and underlined its role as an attractive drug target in vitro and in vivo. MELK was significantly overexpressed in MM patients of the GEP-defined PR subgroup and associated with several genes implicated in cell cycle progression, especially at the G2/M phase. Interestingly, we also observed the overexpression of $M E L K$ at relapse and in PCL, pointing to the reported acquisition of a proliferative character post anti-myeloma therapy. ${ }^{10}$ This finding underlines the association between MELK and aggressive disease. Moreover, the upregulation of MELK or the selection of $M E L K^{\text {high }} \mathrm{MM}$ cells during the course of the disease suggests that a broad patient population could benefit from OTSSP167 treatment compared to a relatively small fraction of patients at baseline (approximately $15 \%$ of patients are categorized into the PR subgroup at diagnosis $\left.{ }^{38}\right)$. These initial data, therefore, strongly supported pre-clinical testing of MELK as a novel drug target for high-risk MM.

The targeting of MELK with specific shRNA significantly reduced the growth of HMCLs. Hence, we studied the impact of pharmacological inhibition of MELK using OTSSP167, currently under investigation in several preclinical and clinical studies. OTSSP167 impairs the autophosphorylation of MELK leading to a subsequent degradation and loss of endogenous MELK protein. ${ }^{31,39}$ Accordingly, we observed a dose-dependent decrease of MELK protein levels 24 hours posttreatment and significant anti-MM activity. This is in line with a recent report demonstrating potent anti-myeloma activity of OTSSP167 in a panel of MM cell lines in vitro. ${ }^{32}$ Our findings corroborate the impact of OTSSP167 on the viability of myeloma cells in mono- and co-culture, demonstrate potent activity in primary $\mathrm{MM}$ cells from high-risk patients and also validated the impact of OTSSP167 on tumor propagating cells using an independent (colony formation) assay. We also demonstrated that the induction of apoptosis coincides with the depolarization of the mitochondrial membrane and loss of MCL-1, a key anti-apoptotic protein in HMCLs. ${ }^{40}$ This corresponds with recent findings demonstrating MCL-1 protein synthesis in a MELK-dependent manner. ${ }^{41}$ The rapid loss of MCL-1 might also explain the adverse effects of combining OTSSP167 with PIs. ${ }^{42}$ In contrast, treatment with OTSSP167 in combination with IMiDs and dexamethasone demonstrated strong synergistic activity and therefore proved the applicability and ben- 
efit of this drug in combination with established anti-MM agents. The strong synergism observed with lenalidomide/pomalidomide plus dexamethasone suggests that OTSSP167 might even enhance the activity of this backbone regimen. OTSSP167 was shown to specifically target MELK in different pre-clinical in vivo models without the induction of severe events. ${ }^{25,31,34}$ Two phase I studies assessing the safety and bioavailability of OTSSP167 have recently been completed (results not published); two additional studies in breast cancer and hematological malignancies are currently ongoing. In the case of no severe toxicities, OTSSP167 should be strongly considered for clinical testing in $\mathrm{MM}$, especially due to its availability as an oral agent and its positive impact on the activity of established myeloma drugs.

Significantly, the anti-myeloma activity of OTSSP167 was confirmed in vivo. We observed a dose-dependent reduction of tumor growth in the murine 5TGM.1 MMmodel. Moreover, treatment displayed a significant increase in the well-being of mice even at sub-optimal anti-myeloma activity. The potent in vivo activity of OTSSP167 confirmed the role of MELK as a novel target in the presence of stromal support, and suggests that OTSSP167 inhibits tumor initiating cells in vivo. The effective targeting of myeloma stem cells was reported by blocking the MELK-associated factor EZH2. ${ }^{33}$ Thus, MELK inhibition might exert its anti-myeloma activity via affecting key players of MM pathophysiology linked to tumor propagation and high-risk disease.

Recent work in glioblastoma revealed a central role for MELK in the regulation of FOXM1-mediated cellular proliferation in a PLK1-dependent manner and EZH2-mediated irradiation resistance. ${ }^{19,30}$ In addition, OTSSP167 was recently shown to target DEPDC1. ${ }^{31}$ These genes were previously described as therapy targets in MM and reported to be associated with high proliferation and/or poor survival. $12,13,15,16,37$ We demonstrated a significant correlation of MELK with all four candidate genes and confirmed the negative impact of these genes on the outcome of myeloma patients. Notably, the overexpression of at least three out of five proliferation-associated high-risk genes was required to unfold their poor prognostic role. This is a key finding of the current study as it clearly demonstrates that it is not a single gene, but rather networks of closely interconnected genes, which drive aggressive disease.

Importantly, the targeting of MELK affected the protein synthesis of all other high-risk genes. Of note, OTSSP167 reduced the gene expression levels of PLK1 and EZH2 but not those of DEPDC1 and FOXM1. In line with this observation, a recent study demonstrated that MELK stabilizes DEPDC1 at the protein level via phosphorylation without affecting DEPDC1 transcription. ${ }^{31}$ The stable expression of FOXM1 at the mRNA level, despite modulation of MELK, is also in line with a previous report. ${ }^{19}$ Moreover, two additional studies demonstrated a decrease of FOXM1 protein expression post OTSSP167 treatment, but did not provide data about FOXM1 mRNA levels. ${ }^{20,32}$ Given that MELK stimulates FOXM1 activation in a PLK1-dependent manner, we hypothesize two pathways that lead to the observed loss of FOXM1 protein expression. First, rapid downregulation of PLK1 (evident 6 hours posttreatment) likely results in reduced FOXM1 activation. Second, FOXM1 is required for mitotic progression, and a loss of activity likely contributes to the observed arrest of MM cells at the G2/M stage of the cell cycle as well as a reduced expression of MELK-FOXM1 downstream target genes such as EZH2. ${ }^{30,43}$ As FOXM1 undergoes proteasomal degradation upon mitotic arrest, a process accelerated by FOXM1 SUMOylation, ${ }^{43}$ we believe that this explains the observed discrepancy between mRNA and protein levels. However, based on recent findings, we cannot exclude potential MELK-independent (off-target) effects of OTSSP167 on these cell cycle-associated genes, ${ }^{44}$ hence further research efforts are required to reveal the exact sequence of OTSSP167 mediated anti-tumor mechanisms and the hierarchy of the MELK-associated gene network.

In silico analysis placed MELK in a network with strong enrichment for key genes of the GEP-defined PR subgroup (DEPDC1, EZH2, FOXM1, NEK2, CCNB1, TOP2A, PRC1, CCNB2, and BIRC5). This suggests that MELK is an important orchestrator of a whole set of proliferative network genes, and blocking MELK appears to represent a promising future strategy to target proliferation-associated myeloma. Independent confirmation of this assumption was obtained from a recent in silico analysis of 645 patients treated within the CoMMpass trial. This study revealed MELK as top driver of a cell cycle-associated pathway in high-risk MM. ${ }^{45}$ Although further studies are needed in order to decipher the exact interaction network and hierarchy of MELK with other high-risk genes, the study herein highlights the strong relation of MELK with proliferation-associated genes and its role as a potential drug target for this group of patients.

Taken together, our data reveal MELK as a novel prognostic marker of proliferation-associated high-risk myeloma and an attractive drug target in MM. The targeting of MELK demonstrated potent anti-myeloma activity, enhanced the activity of IMiDs and dexamethasone, and impaired tumor propagating cells. Furthermore, we demonstrated a strong relationship between MELK and proliferation as well as other proliferation-associated highrisk genes. This suggests that MELK, in conjunction with other high-risk genes, plays an essential role in the regulation of the proliferative phenotype of $\mathrm{MM}$ and that selective targeting of MELK could impair a whole network of central drivers of proliferation-associated MM. These results therefore warrant further investigation into the role of MELK in myeloma and support the clinical testing of OTSSP167 in high-risk MM.

\section{Acknowledgments}

The authors would like to thank Waltraud Scherbler, Katharina Postel, Isrun Bolomsky, Sophie Dubois and the GIGA flow cytometry platform for excellent technical assistance.

\section{Funding}

This study was supported by the Austrian Forum against Cancer, the University of Liège and the Fonds National de la Recherche Scientifique (Belgium). RH and JM are Télévie researchers. JC is a post-doctorate clinical specialist funded by the Belgian Foundation against Cancer. 


\section{References}

1. Kumar SK, Dispenzieri A, Lacy MQ, et al. Continued improvement in survival in multiple myeloma: changes in early mortality and outcomes in older patients. Leukemia. 2014:28(5):1122-1128.

2. Barlogie B, Mitchell A, van Rhee F, et al. Curing myeloma at last: defining criteria and providing the evidence. Blood. 2014;124(20):3043-3051

3. Weinhold N, Heuck CJ, Rosenthal A, et al. Clinical value of molecular subtyping multiple myeloma using gene expression profiling. Leukemia. 2016;30(2):423-430.

4. Sonneveld P, Avet-Loiseau H, Lonial S, et al. Treatment of multiple myeloma with highrisk cytogenetics: a consensus of the International Myeloma Working Group. Blood. 2016;127(24):2955-2962.

5. Paiva B, Almeida J, Pérez-Andrés $M$, et al. Utility of flow cytometry immunophenotyping in multiple myeloma and other clonal plasma cell-related disorders. Cytometry B Clin Cytom. 2010;78(4):239-252.

6. Paiva B, Gutiérrez NC, Rosiñol L, et al. High-risk cytogenetics and persistent minimal residual disease by multiparameter flow cytometry predict unsustained complete response after autologous stem cell transplantation in multiple myeloma. Blood. 2012;119(3):687-691.

7. Paiva B, Vídriales M-B, Rosiñol L, et al. A multiparameter flow cytometry immunophenotypic algorithm for the identification of newly diagnosed symptomatic myeloma with an MGUS-like signature and long-term disease control. Leukemia. 2013;27(10):2056-2061.

8. Shaughnessy JD, Zhan F, Burington BE, et al. A validated gene expression model of high-risk multiple myeloma is defined by deregulated expression of genes mapping to chromosome 1 . Blood. 2007;109(6):2276-2284.

9. Hose D, Rème $T$, Hielscher $T$, et al. Proliferation is a central independent prognostic factor and target for personalized and risk-adapted treatment in multiple myeloma. Haematologica. 2011;96(1):8795.

10. Zhan F, Huang Y, Colla S, et al. The molecular classification of multiple myeloma. Blood. 2006;108(6):2020-2028.

11. Hose $\mathrm{D}$, Rème $\mathrm{T}$, Meissner $\mathrm{T}$, et al. Inhibition of aurora kinases for tailored risk-adapted treatment of multiple myeloma. Blood. 2009;113(18):4331-4340.

12. McMillin DW, Delmore J, Negri J, et al. Microenvironmental influence on pre-clinical activity of polo-like kinase inhibition in multiple myeloma: implications for clinical translation. PLoS One. 2011;6(7):e20226.

13. Evans RP, Dueck G, Sidhu R, et al. Expression, adverse prognostic significance and therapeutic small molecule inhibition of Polo-like kinase 1 in multiple myeloma. Leuk Res. 2011;35(12):1637-1643.

14. Noll JE, Vandyke K, Hewett DR, et al. PTTG1 expression is associated with hyperproliferative disease and poor prognosis in multiple myeloma. J Hematol
Oncol. 2015;8:106

15. Kassambara A, Schoenhals M, Moreaux J, et al. Inhibition of DEPDC1A, a bad prognostic marker in multiple myeloma, delays growth and induces mature plasma cell markers in malignant plasma cells. PloS One. 2013;8(4):e62752

16. Gu C, Yang Y, Sompallae R, et al. FOXM1 is a therapeutic target for high-risk multiple myeloma. Leukemia. 2016;30(4):873-882.

17. Ganguly R, Hong C, Smith L, Kornblum HI, Nakano I. Maternal embryonic leucine-zipper kinase: key kinase for stem cell phenotype in glioma and other cancers. Mol Cancer Ther. 2014;13(6):1393-1398.

18. Nakano I, Paucar AA, Bajpai R, et al. Maternal embryonic leucine zipper kinase (MELK) regulates multipotent neural progenitor proliferation. J Cell Biol. 2005; 170(3):413-427.

19. Joshi K, Banasavadi-Siddegowda Y, Mo X, et al. MELK-dependent FOXM1 phosphorylation is essential for proliferation of glioma stem cells. Stem Cells. 2013;31(6):1051-1063.

20. Alachkar $\mathrm{H}$, Mutonga MB, Metzeler $\mathrm{KH}$, et al. Preclinical efficacy of maternal embryonic leucine-zipper kinase (MELK) inhibition in acute myeloid leukemia. Oncotarget. 2014;15(5):12371-12382.

21. Marie SKN, Okamoto OK, Uno M, et al. Maternal embryonic leucine zipper kinase transcript abundance correlates with malignancy grade in human astrocytomas. Int J Cancer. 2008;122(4):807-815.

22. Pickard MR, Green AR, Ellis IO, et al. Dysregulated expression of Fau and MELK is associated with poor prognosis in breast cancer. Breast Cancer Res. 2009;11(4):R60.

23. Kuner R, Fälth M, Pressinotti NC, et al. The maternal embryonic leucine zipper kinase (MELK) is upregulated in high-grade prostate cancer. J Mol Med (Berl). 2013; 91(2):237-248.

24. Du T, Qu Y, Li J, et al. Maternal embryonic leucine zipper kinase enhances gastric cancer progression via the FAK/Paxillin pathway. Mol Cancer. 2014;13:100

25. Li S, Li Z, Guo T, et al. Maternal embryonic leucine zipper kinase serves as a poor prognosis marker and therapeutic target in gastric cancer. Oncotarget. 2016;7(5):62666280

26. Xia H, Kong SN, Chen J, et al. MELK is an oncogenic kinase essential for early hepatocellular carcinoma recurrence. Cancer Lett. 2016;383(1):85-93

27. Nakano I, Masterman-Smith M, Saigusa K, et al. Maternal embryonic leucine zipper kinase is a key regulator of the proliferation of malignant brain tumors, including brain tumor stem cells. I Neurosci Res. 2008:86(1):48-60.

28. Nakano I, Joshi K, Visnyei K, et al. Siomycin A targets brain tumor stem cells partially through a MELK-mediated pathway. Neuro Oncol. 2011;13(6):622-634.

29. Wang Y, Lee Y-M, Baitsch L, et al. MELK is an oncogenic kinase essential for mitotic progression in basal-like breast cancer cells. Elife. 2014;3:e01763.

30. Kim S-H, Joshi K, Ezhilarasan R, et al.
EZH2 protects glioma stem cells from radiation-induced cell death in a MELK/FOXM1-dependent manner. Stem Cell Rep. 2015;4(2):226-238.

31. Chung S, Kijima K, Kudo A, et al. Preclinical evaluation of biomarkers associated with antitumor activity of MELK inhibitor. Oncotarget. 2016; 7(14):1817118182.

32. Stefka AT, Park J-H, Matsuo Y, et al. Antimyeloma activity of MELK inhibitor OTS167: effects on drug-resistant myeloma cells and putative myeloma stem cell replenishment of malignant plasma cells. Blood Cancer J. 2016;6(8):e460

33. Zeng D, Liu M, Pan J, et al. Blocking EZH2 methylation transferase activity by GSK126 decreases stem cell-like myeloma cells. Oncotarget. 2017;8(2):3396-3411.

34. Chung S, Suzuki H, Miyamoto $T$, et al Development of an orally-administrative MELK-targeting inhibitor that suppresses the growth of various types of human cancer. Oncotarget. 2012;3(12):1629-1640

35. Bolomsky A, Schlangen K, Schreiner W Zojer N, Ludwig H. Targeting of BMI-1 with PTC-209 shows potent anti-myeloma activity and impairs the tumour microenvironment. J Hematol Oncol. 2016:9:17.

36. Heusschen R, Muller J, Binsfeld M, et al SRC kinase inhibition with saracatinib limits the development of osteolytic bone disease in multiple myeloma. Oncotarget. 2016;7(21):30712-30729.

37. Hernando H, Gelato KA, Lesche R, et al. EZH2 inhibition blocks multiple myeloma cell growth through upregulation of epithelial tumor suppressor genes. Mol Cancer Ther. 2016;15(2):287-298.

38. Weinhold N, Heuck C, Rosenthal A, et al. The clinical value of molecular subtyping multiple myeloma using gene expression profiling. 2016;30(2):423-430.

39. Inoue $\mathrm{H}$, Kato $\mathrm{T}$, Olugbile $\mathrm{S}$, et al. Effective growth-suppressive activity of maternal embryonic leucine-zipper kinase (MELK) inhibitor against small cell lung cancer Oncotarget. 2016;7(12):13621-13633.

40. Gong J-N, Khong T, Segal D, et al Hierarchy for targeting pro-survival BCL2 family proteins in multiple myeloma: pivotal role of MCL1. Blood. 2016; 128(14):1834-1844.

41. Wang Y, Begley M, Li $\mathrm{Q}$, et al. Mitotic MELK-eIF4B signaling controls protein synthesis and tumor cell survival. Proc Nat Acad Sci U S A. 2016;113(35):9810-9815

42. Podar K, Gouill SL, Zhang J, et al. A pivotal role for Mcl-1 in Bortezomib-induced apoptosis. Oncogene. 2008;27(6):721-731.

43. Myatt SS, Kongsema M, Man CW, et al. SUMOylation inhibits FOXM1 activity and delays mitotic transition. Oncogene. 2014; 33(34):4316-4329

44. Ji W, Arnst C, Tipton AR, et al. OTSSP167 abrogates mitotic checkpoint through inhibiting multiple mitotic kinases. PLoS One. 2016;11(4):e0153518.

45. Gruber F, Keats JJ, McBride K, et al Bayesian network models of multiple myeloma: drivers of high risk and durable response. 2016; 128(22):4406-4406. 